\title{
Leveraging Wettability Engineering to Develop Three-Layer DIY Face Masks From Low-Cost Materials
}

\author{
Sourav Sarkar ${ }^{1,2} \cdot$ Achintya Mukhopadhyay $^{1,2} \cdot$ Swarnendu Sen $^{1,2} \cdot$ Safwan Mondal ${ }^{1,2} \cdot$ Arnav Banerjee $^{1,2}$. \\ Pranibesh Mandal ${ }^{1,2} \cdot$ Ratna Ghosh $^{1,3} \cdot$ Constantine M. Megaridis ${ }^{4} \cdot$ Ranjan Ganguly $^{1,4,5}$
}

Received: 14 April 2020 / Revised: 21 May 2020 / Accepted: 26 May 2020 / Published online: 4 June 2020

(c) Indian National Academy of Engineering 2020

\begin{abstract}
With the rapid spread of COVID-19 worldwide, the demand for appropriate face masks in the market has also skyrocketed. To ease strain on the supply of masks to the essential healthcare sector, it has become imperative that ordinary people rely more on home-made masks that can be easily put together using commonly available materials, while at the same time performing reasonably at arresting the ingress or egress of airborne droplets. Here, we propose a simple do-it-yourself (DIY) method for preparing a three-layered face mask that deploys two hydrophobic polypropylene nonwoven layers interspaced with a hydrophilic cellulosic cloth. The first hydrophobic layer, facing the user, allows high-momentum droplets (e.g., expelled by a sneeze or cough) to pass through and get absorbed in the next hydrophilic layer, thereby keeping the skin in contact with the mask dry and comfortable. The third (outermost) hydrophobic layer prevents penetration of the liquids from the middle layer to the outside, and also arrests any airborne droplets on its exterior. Simple tests show that our masks perform better in arresting the droplet transmission as compared to surgical masks available in the market.
\end{abstract}

Keywords COVID-19 $\cdot$ Face mask $\cdot$ Wettability engineering $\cdot$ Nonwoven $\cdot$ Droplet transport

\section{Introduction}

At the writing of this manuscript, the deadly Novel Coronavirus is on a rampage, having infected nearly 4.2 million people worldwide already, claiming about $6 \%$ of them. While airborne droplets, emitting from the cough and

Electronic supplementary material The online version of this article (https://doi.org/10.1007/s41403-020-00115-9) contains supplementary material, which is available to authorized users.

Ranjan Ganguly

ranjan.ganguly@jadavpuruniversity.in

1 Center for Development of Appropriate Social Technologies (CAST), Jadavpur University, Kolkata, India

2 Mechanical Engineering Department, Jadavpur University, Kolkata, India

3 Instrumentation and Electronics Engineering Department, Jadavpur University, Kolkata, India

4 Mechanical and Industrial Engineering Department, University of Illinois at Chicago, Chicago, USA

5 Power Engineering Department, Jadavpur University, Kolkata, India sneeze of the infected population is directly implicated for the spread of the disease, details on the risks associated with different human activities like sneezing, coughing and even talking aloud for transmission of this deadly virus are still emerging (Beans 2020). As a consequence, advisories on use of masks, especially by non-affected population outside the care-giving fraternity have seen significant revisions both at the national and international levels. Although WHO advisory ( https://www.who.int/emergencies/diseases/ novel-coronavirus-2019/advice-for-pub1/when-and-how-touse-masks 2020) had earlier recommended the use of N95 face masks for all healthcare professionals and the patients, it discouraged the use of N95 masks by common people - primarily to ease the pressure on N95 demand, so those who really need the masks can get them- the latest advisory (World Health Organization 2020) has listed benefits of mask use even by the unaffected population. Similarly, the Indian Ministry of Health and Family Welfare has modified its earlier advisory (Ministry of Health and Family Welfare 2020a) for the use of masks by only affected individuals and frontline caregivers, to recommend use of ordinary (i.e., non-N95 grade) homemade masks, made of cloth, by every individual (Ministry of Health and Family Welfare 2020b). 
Thus, there is now a general urgency for widespread production and use of protective masks to reduce risk of both infection by asymptomatic carriers and community-level infection of healthy individuals. In the present situation, it is difficult to supply masks to each and every corner of a large country like India. Moreover, the high price of some available masks is prohibitive for a large a section of our population, thus preventing the widespread use of masks.

A crucial piece of information for designing protective masks and assessing their efficacy is the fluid dynamic data related to coughing and sneezing, namely, volume flow rate of air ejected along with number density, size and velocity distributions of the ejected droplets and aerosols. Both these factors play determining roles in the trajectory of the droplets and their longevity as air-borne particles. Although such detailed studies in the context of COVID-19 virus transmission are, expectedly, scarce in the peer-reviewed open literature, similar studies are available in the context of other air-borne infectious diseases, like influenza and earlier versions of corona virus, like SARS and MERS. Highspeed flow visualization studies have shown that during violent respiratory events, like sneezing and coughing, the pathogen-carrying droplets of diameter $10 \mu \mathrm{m}$ may remain suspended even at several meters away from the source (Bouroiba et al. 2014). Moreover, such small droplets, from the cough or sneeze cloud can be re-suspended by the ambient air currents having velocities of the order of $1 \mathrm{~cm} / \mathrm{s}$. A study on the fluid dynamics of cough (Gupta et al. 2009) has shown that the instantaneous volume flow rate of air could be represented as a combination of gamma functions, whose parameters vary with the medical condition of an individual. A typical cough was observed to generate peak flow rates in the range 3-8.5 $\mathrm{l} / \mathrm{s}$ in males and $1.6-6 \mathrm{l} / \mathrm{s}$ in females, while the total volume exhaled was $400-1600 \mathrm{ml}$ in males and $250-1250 \mathrm{ml}$ in females. It was found that sequential coughs were combinations of single coughs. The first cough was found to be identical to a single cough, while the second one was a scaled-down version of the first. Yang et al. (2007) reported that the average droplet size distribution was $0.58-5.42 \mu \mathrm{m}$ and $82 \%$ of the droplets were in the range $0.74-2.12 \mu \mathrm{m}$. Thus, face masks have dual challenges of preventing liquid penetration for high-velocity droplets from inside to outside during cough and sneeze, and also the ingress of suspended droplets from the ambient.

Here we discuss an effort by CAST, Jadavpur University, to develop a simple procedure for making a three-layer mask from low cost, readily available materials. The choice of materials, which, in this case are readily available in a house or in the neighborhood, is important since the supply of raw materials is an issue in these days of lock-down or restricted mobility. We ensured that by using these materials, the effectiveness of the mask has not been sacrificed. The process is simple enough to make a mask on one's own in any of the available DIY designs templates chosen by the individual. At the same time, we paid particular attention to the salient functional requirements of the mask: (1) compared to the N95 masks (which is strictly recommended for acute patients and healthcare professionals), the masks are more breathable, so that people can wear it over longer duration without serious discomfort; this implies that the pore size is larger than the N95 filters; (2) the three layers should prevent escape of droplets as the wearer sneezes through it, and at the same time, the innermost layer of the mask should remain dry to ensure comfort; (3) the mask should prevent ingress of droplets from the ambient during inhalationboth through the fabric and the gap between the face and the mask; (4) the mask is easy to sanitize/disinfect for re-use.

\section{Materials and Methods}

Keeping in view the above requirements, the proposed mask is made of three layers of fabric - the innermost one, which is in contact with the person wearing it, the intermediate one, and the outermost layer. The innermost and outermost layers are made of a commonly-available, nonwoven, polypropylene fabric, which intrinsically has a mildly hydrophobic nature. Presently, we have proposed the cloth carry bags (which are often used as a substitute for plastic shopping bags) as a quick option. The intermediate layer is made up of cotton/cellulosic fibers that are intrinsically hydrophilic. A material which can be used is a cloth duster or a cotton towel (the ubiquitous Bengali gamchha) for this layer. One could also use old cotton cloth for this layer. Figure 1a shows the sequential arrangement of the three layers. Inset (a1) shows a magnified view (taken through a standard cellphone camera and a DIY-Kids' Science kit microscope) of the polypropylene layer. The layer comprises of loosely-spun polypropylene (PP) fibers that are held together by periodic thermal stamps. Zoomed-in views of the cellulosic (C) hydrophilic layer (inset a2) show the woven structure of the intermediate cellulosic fibers. When a $\sim 5 \mu$ size water droplet was dispensed on the polypropylene fabric and imaged using again a science toolbox USB camera (CARPRIE $5.5 / 7 \mathrm{~mm}$ mini waterproof endoscope camera), it displayed a static contact angle of $\theta_{s}=120^{\circ} \pm 3^{\circ}$ (inset a3) and advancing contact angle of $\theta_{\text {adv }}=131^{\circ} \pm 2^{\circ}$ (inset a4). Since the PP fabric is intrinsically hydrophobic, no apparent degradation of the surface wettability is noticed upon several (tested up to 5) cycles of washing with a standard detergent, followed by adequate rinsing and sun-drying. When viewed under the in-house goniometer, the observed change in the static and advancing contact angles remained below $\pm 2^{\circ}$ (based on 5 readings), which was within the error bars. The cellulosic layer exhibited rapid wicking (see the video played at 1/4th speed in (https://www.dropbox.com/s/1h30prgk221rtaz/ESI\%20Ved 

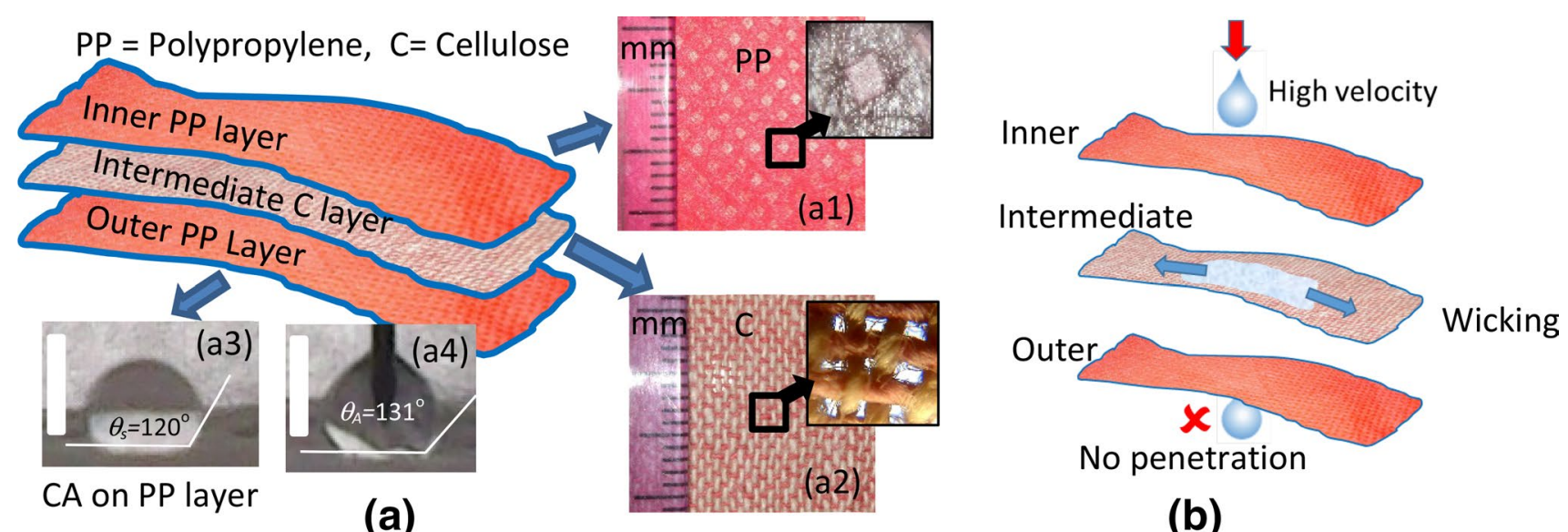

(b)

Fig. 1 a The three-layer structure of the mask, comprising of an innermost, non-woven polypropyelene (PP) layer, an intermediate cellulosic (C) layer and an outermost polypropylene (PP) layer. Insets (a1) and (a2) show the zoomed-in views of the fabrics, and the fiber

io.MP4dl=0 2020), thus rendering any contact angle measurement impossible.

\section{Principle of Droplet Penetration}

The combination of stacked hydrophobic-hydrophilichydrophobic layers typically follows the principle of liquid diode, which is characterized by preferential transport of liquid droplets (Mates et al. 2014; Chen et al. 2012). A porous substrate having a hydrophobic top and an underlying hydrophilic layer will allow a liquid droplet to pass through the top and spread at the bottom layer when the droplet dispensed on the top has a momentum and/or capillary pressure exceeding the capillary resistance of the hydrophobic porous material (the hydrohead) (Chatterjee et al. 2018). A droplet impacting on a porous substrate with a velocity $U$ will penetrate the substrate if its dynamic pressure $\left(p_{d} \sim \rho U^{2}\right)$ exceeds the resisting capillary pressure $\left(\sim \sigma \cos \theta_{\text {adv }} / d_{\text {pore }}\right)$, where $\rho$ denotes the liquid density $\left(\sim 1000 \mathrm{~kg} / \mathrm{m}^{3}\right), \sigma$ the liquid surface tension $(0.07 \mathrm{~N} / \mathrm{m}$ for water $)$, and $d_{\text {pore }}$ the equivalent pore diameter $(\sim 100 \mu \mathrm{m}$ for the polypropylene bag materials; see Fig. 1a1), the penetration velocity of $\sqrt{ }\left(\sigma \cos \theta_{\text {adv }} / \rho d_{\text {pore }}\right) \sim 0.68 \mathrm{~m} / \mathrm{s}$. Typical droplet velocity close to the mouth during sneezing and coughing, which exceeds $\sim 6 \mathrm{~m} / \mathrm{s}$, (Nishimura et al. 2013) implies that when a person wearing this mask sneezes, droplets penetrate the innermost polypropylene layer and get absorbed in the intermediate layer. Further penetration through the intermediate layer is prevented due to the immediate wicking action of the hydrophilic cellulosic material. Moreover, the combination of the hydrophilic intermediate layer followed by the hydrophobic outermost one behaves in the non-penetrative direction of the liquid diode (Mates et al. 2014; Chatterjee laying. Other insets show the static contact angle, $\theta_{s}$ (a3) and advancing contact angle $\theta_{\text {adv }}(\mathrm{a} 4)$ on the PP layer (vertical scale bars measure $2 \mathrm{~mm}$ ). b Schematic of the liquid droplet retention in the threelayer fabric of the face mask

et al. 2018) thus preventing escape of droplets to the ambient around the wearer. The same physics also prevents backflow of the soaked liquid from the intermediate layer to the innermost polypropylene layer, thus keeping the contact surface (next to the skin) dry and ensuring human comfort and hygiene. Any droplet suspended in the ambient has a possibility of impinging on the outermost hydrophobic layer during inhaling phase. However, these droplets will, in general be arrested on the hydrophobic layer since the droplet velocities will be below the prerequisite penetration velocity.

\section{Fabrication of the Mask}

Once the three-layer fabric assembly has been prepared by stitching them together, the mask can be made following any suitable mask-making template available online. We have used one method to make a prototype (Fig. 2) using a common sewing machine. In the absence of a sewing machine, the mask can be hand-stitched as well. Care has been taken while adopting the correct template, so that the mask has the best possible conformity to the face, and the leakage through gaps between the mask and the skin is minimal. The students' group has played a key role in executing the quick fabrication. The group has also prepared videos (https $: / /$ www.youtube.com/watchv=TBOqofEaoOct=127s2020) to disseminate the procedure among all people. On one hand, any organization can take up the procedure to make it and supply in large numbers; on the other hand, any person can make it for his/her own protection. Feedbacks from the individual users have also been positive so far (for a dynamically updated feedback summary, see: https://www.dropbox.com/ s/8z1mnbyy5hfdyb7/User\%20Feedback.pdf?dl=0). 


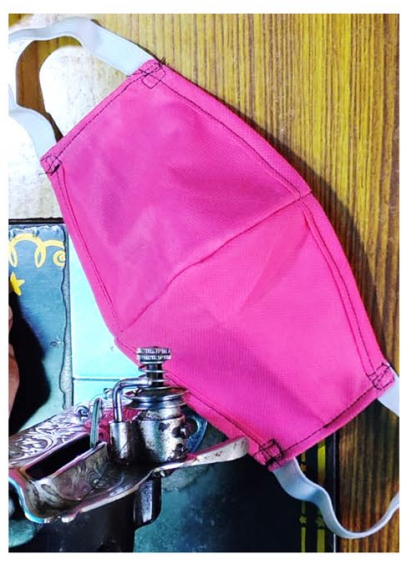

Reckoning the raw material cost at the retail market and labor costs, the production cost of the mask was estimated to be Rs. $4.5-6$ per piece, which can go down by about $50 \%$ if the masks are made in large quantities. It is important to note that several improvements in the layout of the mask design and the template is possible (see the Electronic Supplementary Information Figure R1 for another design), which is left as a future exercise. The technology has already been picked up by MSME entities, with minor modifications in the physical layout, for mass production (https://www.sulek haink.co.in, Product brochure 2020, page 5).

\section{Performance Test of the Mask}

The technical specifications of the readily available fabric layers were not available (the porosity and the liquid spreading coefficients of gamcha are not reported) and could not be measured sans the access to the university laboratory facilities due to the nationwide lockdown. Therefore, the most important part of the development was testing the threelayer fabric for a near-real-life situation. A quick, in-house experiment was conducted to verify the effectiveness of the mask in arresting the droplets generated from sneezing of the person wearing a mask (see Fig. 3a). A finely-divided spray (photograph in Fig. 3a) of water droplets was impacted from close proximity $(\sim 50 \mathrm{~mm})$ on the innermost polypropylene layer; each puff from the hand-sprayer dispensed $\sim 112 \mu \mathrm{l}$ of water (This is comparable with the typical volume $(\sim 75 \mu \mathrm{l})$ of fluid ejected in a single cough) (Sze To et al. 2009; Zhang 2017). Any trace of penetration from the outermost polypropylene layer was visually inspected by holding the mask against a mirror-finish surface during the spray, and checking the mirror surface and the outer surface of the mask for traces of liquid after the spray-test. The sprinkled water droplets were found to cross the innermost polypropylene layer, keeping its exposed (to the skin) surface dry. The middle cotton layer was found (after spraying dyed water and then cutting open the mask) to retain the liquid droplets by wicking (shown by the spread of dyed water in Fig. 3b). No noticeable trace of moisture on the mirror-finish test plate was observed on the outermost side (Fig. 3c) even after 30 puffs of spray (i.e., $3.6 \mathrm{ml}$ of liquid), indicating that the second polypropylene layer prevented droplet transmission to its outer exposed surface. Droplet prevention in the reverse flow was also arrested by this design. When a finely divided mist was released on the outermost polypropylene layerthis time not with high impact, since they emulate droplets suspended in ambient air - the droplets could not penetrate the hydrophobic outer layer. The momentum of the droplets in this case could not overcome the capillary pressure needed to transport through the pores of the polypropylene layer. Thus, they stayed on the outermost surface and eventually evaporated. 

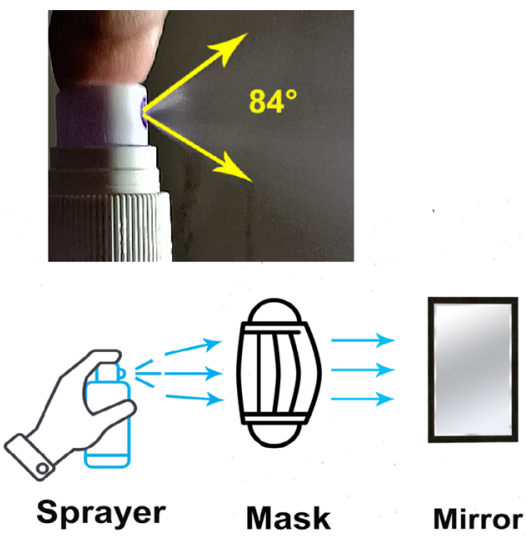

(a)

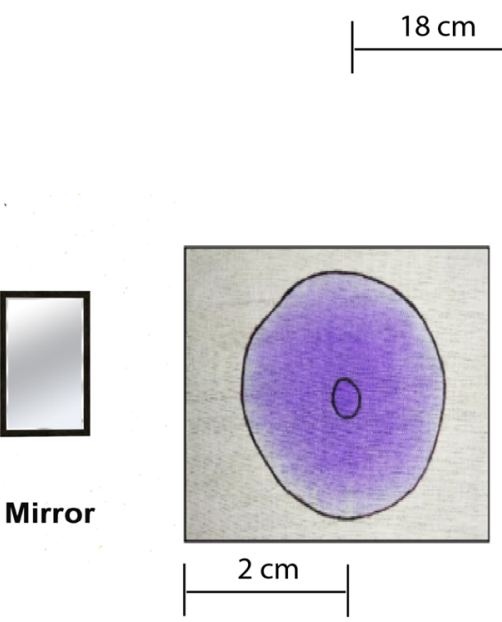

(b)

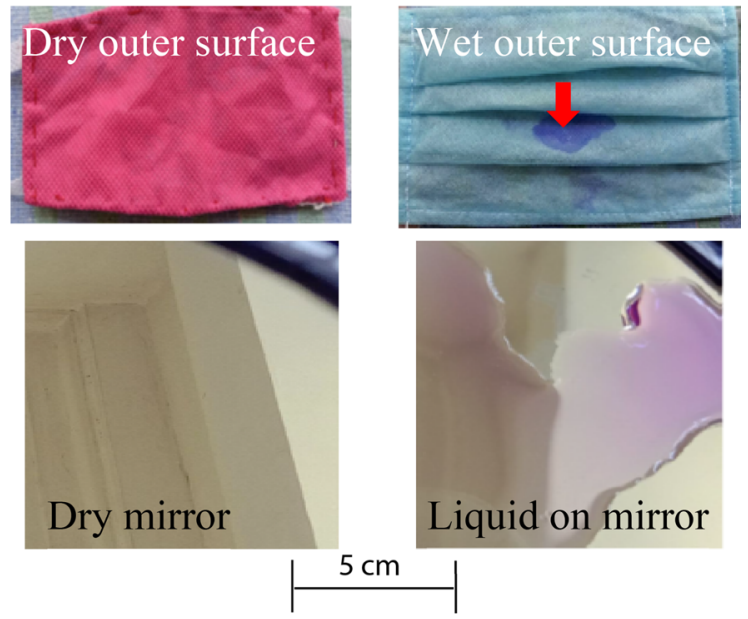

(c) (d)
Fig. 3 Performance test of the mask: a the testing setup (the photograph shows the spray cone angle), $\mathbf{b}$ wicking in the middle cotton layer after spraying dyed water and then cutting open the mask. c

Hence, the mask is also expected to prevent ingress of airborne liquid droplets during inhalation. The performance of the mask did not degrade even after multiple (up to 5 times) washing cycles. It was also verified that a person could breathe comfortably after wearing this mask. Further quantitative experimentation (e.g. pressure drop across the mask layers) can be carried out once the University laboratories become accessible again. A comparison was also made with a common triple-layer surgical mask available in the market. Figure $3 \mathrm{~d}$ shows the result of spray penetration test, where traces of moisture were found on the mirror surface, as well as on the exterior of the mask, under similar conditions of testing, indicating that the three-layer surgical mask failed (to prevent droplet penetration) upon 30 puffs of spray.

\section{Conclusion}

A low-cost DIY face mask has been prepared using three layers of porous fabric where a hydrophilic cellulosic intermediate layer is sandwiched between two moderately hydrophobic layers of non-woven commercially-available polypropylene. The combination of the innermost and the middle layer allow high-momentum droplets to penetrate the hydrophobic layer and get absorbed on the hydrophilic layer, while the outermost hydrophobic layer prevents any escape of droplets to the surroundings of the wearer. Reverse flow from the outside through the outermost polypropylene layer, or from the middle layer to the innermost layer is also prevented. Simple test using a garden sprayer shows the ability of the mask in restricting through transmission
No trace of water found to penetrate the our triple-layer mask after impinging 30 puffs of the spray, while (d) a regular two-layer surgical mask from the market showed liquid penetration

of the droplets, either outbound during a sneeze or cough or during inhaling.

Acknowledgements Authors would like to acknowledge Mr. Krishnendu Maji and Mr. Sabyasachi Mondal for preparing the mask and the video of the mask-making procedure, and Mr. Kamalesh Roy of People's Initiative for Technology for providing the estimate of the unit cost of the mask. The authors also acknowledge Master Riddhiman for the images in Fig. 1 with his DIY-Kids' Science kit microscope and the science toolbox USB camera, and the high-speed video of liquid spreading on cellulosic fabric.

\section{References}

Advice on the use of masks in the context of COVID-19, Interim Guidance, World Health Organization, https://www.who.int/publicatio ns-detail/advice-on-the-use-of-masks-in-the-community-durin g-home-care-and-in-healthcare-settings-in-the-context-of-thenovel-coronavirus-(2019-ncov)-outbreak, Posted on April 6, 2020

Advisory on use of Homemade Protective Cover for Face \& Mouth, Ministry of Health and Family Welfare, Government of India, https://www.mohfw.gov.in/pdf/Advisory\&ManualonuseofHo memadeProtectiveCoverforFace\&Mouth.pdf, Posted on April 3, 2020.

Beans C (2020) Fluid dynamics work hints at whether spoken word can spread COVID-19. https://blog.pnas.org/2020/04/fluid-dynamicswork-hints-at-whether-spoken-word-can-spread-covid-19/, Posted on April 7, 2020.

Bouroiba L, Dehandschoewercker E, Bush JWM (2014) Violent expiratory events: on coughing and sneezing. J Fluid Mech 745:537-563

Chatterjee S, Mahapatra PS, Ibrahim A, Ganguly R, Yu L, Dodge R, Megaridis CM (2018) Precise liquid transport on and through thin porous materials. Langmuir 34:2865-2875

Chen H, Cogswell J, Anagnostopoulos C, Faghri MA (2012) A fluidic diode, valves, and a sequential-loading circuit fabricated on layered paper. Lab Chip 12:2909-2913 
Guidelines on use of masks by public, Ministry of Health and Family Welfare, Government of India, https://www.mohfw.gov.in/pdf/ Useofmaskbypublic.pdf, Posted on March 11, 2020

Gupta J, Lin C-H, Chen Q (2009) Flow dynamics and characterization of a cough. Indoor Air 19:517-525

https://www.who.int/emergencies/diseases/novel-coronavirus-2019/ advice-for-public/when-and-how-to-use-masks, Posted on January 29, 2020 (last updated on March 18, 2020).

https://www.youtube.com/watch?v=TBOqofEaoOc\&t=127s, Uploaded on Apr 6, 2020.

Mates JE, Schutzius TM, Qin J, Waldroup DE, Megaridis CM (2014) The Fluid diode: tunable unidirectional flow through porous substrates. ACS Appl Mater Interfaces 6:12837-12843

Nishimura H, Sakata S, Kaga A (2013) A new methodology for studying dynamics of aerosol particles in sneeze and cough using a digital high-vision, high-speed video system and vector analyses. PLoS ONE 8:e80244

See the ESI video: https://www.dropbox.com/s/1h30prgk221rtaz/ ESI\%2520Vedio.MP4?dl=0.
Sze To GN, Wan MP, Chao CYH, Fang L, Melikov A (2009) Experimental study of dispersion and deposition of expiratory aerosols in aircraft cabins and impact on infectious disease transmission. Aerosol Sci Technol 43:466-485

www.sulekhaink.co.in, Product brochure, page 5.

Yang S, Lee GWM, Chen C-M, Wu C-C, Yu K-P (2007) The size and concentration of droplets generated by coughing in human subjects. J Aerosol Med 20:484-494

Zhang B (2017) Aerosol transport by coughing in a depressurized aerosol chamber, Ph.D. Dissertation. New Jersey Institute of Technology, New Jersey

Publisher's Note Springer Nature remains neutral with regard to jurisdictional claims in published maps and institutional affiliations. 